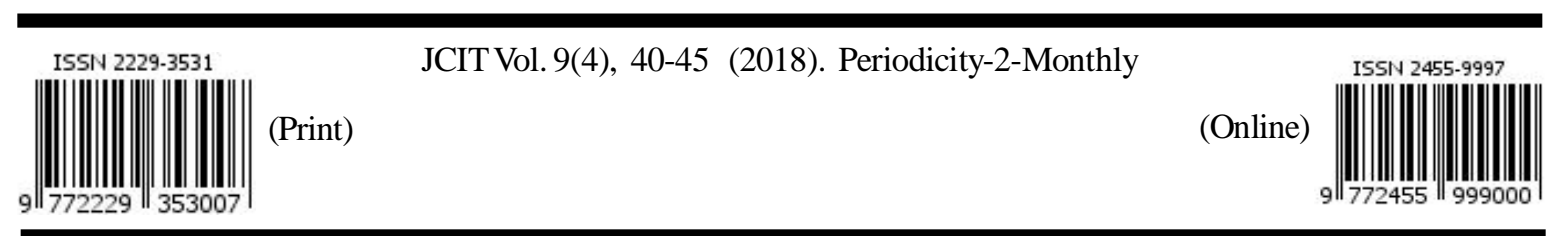

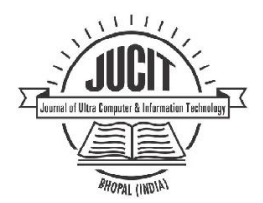

JOURNAL OF COMPUTER \& INFORMATION TECHNOLOGY

An International Open Free Access Peer Reviewed Research Journal of Computer

Science Engineering \& Information Technology

website:- www.compitjournal.org

Estd. 2010

\title{
A Differential Image Compression Method Using Quantiser Mechanism
}

RESHU JAIN, RAJNI KORI and RACHNA DUBEY

\author{
M. Tech Scholar, Department of Computer Science \& Engineering, \\ Lakshmi Narain College of Technology Excellence Bhopal (M.P) (India) \\ Assistant Professor, Department of CSE, Lakshmi Narain College of Technology Excellence, Bhopal (M.P), India \\ Professor \& Head, Department of CSE, Lakshmi Narain College of Technology Excellence, Bhopal (M.P), India \\ Corresponding Author Email: reshuam8421@gmail.com \\ http://dx.doi.org/10.22147/jucit/090401
}

Acceptance Date 14th July 2018,

Online Publication Date 2nd August, 2018

\begin{abstract}
Images include information about human body which is used for different purposes such as medical, security and other plans. Compression of images is used in some applications such as profiling data and transmission systems. Regard to importance of images information, lossless or lossy compression is preferred. Lossless compressions are JPEG, JPEGLS and JPEG2000 are few well-known methods for lossless compression. We will use differential pulse code modulation for image compression with Huffman encoder, which is one of the latest and provides good compression ratio, peak signal noise ratio and minimum mean square error. In real time application which needs hardware implementation, low complex algorithm accelerates compression process. In this paper, the authors use differential pulse code modulation for image compression lossless and near-lossless compression method is introduced which is efficient due to its high compression ratio and simplicity. This method consists of a new transformation method called Enhanced DPCM Transformation (EDT) which has a good energy compaction and a suitable Huffman encoding. After introducing this compression method, it is applied on different images from Corel dataset for experimental results and analysis. Also we compare it with other existing methods with respect to parameter compression ratio, peak signal noise ratio and mean square error.

Key words: Lossless Compression, Image Transformation, Prediction Method, Encoding Technique, DPCM.

\section{Introduction}

In today's world, digital images are being widely used in numerous applications such as internet world, military, intelligence, surveillance, digital copyright applications, etc.

With the increase in use of multimedia type data over the internet. The Image compression plays an important role in storage and communication. Images are being transferred over the Internet and are readily available

for access from any part of the world. The growth of multimedia technology over past decades, the demand for digital information increases dramatically. The advances in technology have made the use of digital images prevent to a large extents. Still images are widely used in application like medical and satellite images. Digital images are comprised of a enormous amount of data. Reduction in the size of the image data for both storing \& transmission of digital images are becoming increasingly important as they find more application $^{1}$.
\end{abstract}

This is an open access article under the CC BY-NC-SA license (https://creativecommons.org/licenses/by-nc-sa/4.0) 
Image compression is a mapping from higher dimensio nal space to a lower dimensional space. Image compression plays an important role in many multimedia application .The basic goal of image compression is to represent an image with minimum number of bits of an acceptable image quality. All image compression algorithms strive to remove statistical redundancy and exploit perceptual irrelevancy while reducing the amount of data as much as possible.

Image: An image is a two-dimensional function that represents a measure of some characteristic such as brightness or colour of a viewed scene. It can be defined as two variable function $f(x, y)$, where for each position $(x, y)$ in the projection plane, $\mathrm{f}(\mathrm{x}, \mathrm{y})$ defines the light intensity at this point.

We have two types of image on the basis of its nature which is given below:

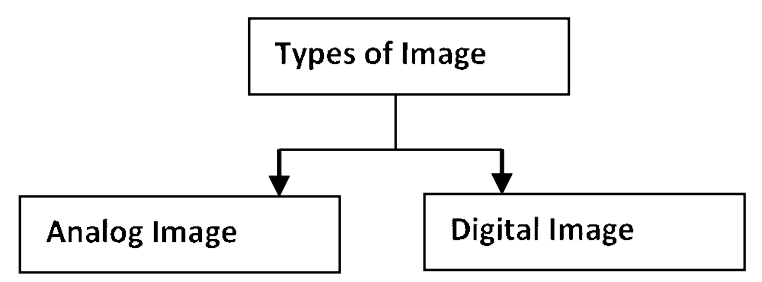

Figure 1: Type of Images [1]

Analog Image: An analog image can be mathematically represented as continuously range of values representing position and intensity. An analog image is characterized by physical magnitude varying continuously in space.eg. An image produced on the screen of CRT monitor is analog in nature ${ }^{2}$.

Digital Image: A digital image is composed of picture element called pixels. Pixels are smallest sample of an image. A pixel represents the brightness at one point.

There is following steps used in conversion from Analog to Digital:

\begin{tabular}{|l|l|}
\hline Analog Image & $\rightarrow$ Sampling $\rightarrow$ Quantisation $\rightarrow$ Digital Image \\
\hline
\end{tabular}

Figure2: Digital Image from Analog Image [2]

\subsection{Purpose of Image Compression Methods and Algorithms :}

The main purpose of image compression is to reduce the size of image data for both storing and transmission. Image compression is mapping from higher dimensional space to a lower dimensional space.

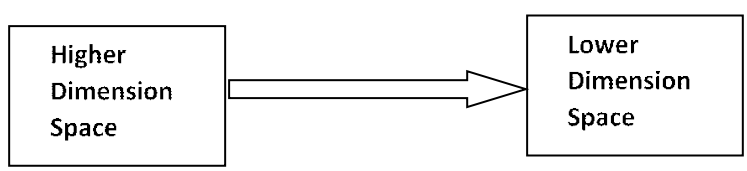

Figure 3: Image Compression Purpose [3]

\section{DESCRIPTION OF IMAGE COMPRESSION TECHNIQUES :}

2.1 Overview :

Image compression is a technique by which we represent an image with minimum number of bits of an acceptable image quality. In other words we can say that image compression is done to reduce the amount of image data as soon as possible ${ }^{17}$.

\subsection{Classification of image compression :}

Image compression schemes can be broadly classified in two types:

Lossless Compression (Reversible Compression): In lossless compression, the image after compression and decompression is identical to the original image and every bit of information is preserved during the decomposition process. The reconstructed image after compression is an exact replica of original one.

Lossy Compression (Irreversible Compression): In lossy compression, the reconstructed image contains degradations with respect to the original image. Hence, perfect reconstruction of the image is sacrificed by the elimination of some amount of redundancies.

\subsection{Image Compression Scheme:}

The source encoder and decoder pair is commonly referred as source code module, whereas the channel encoder and decoder pair is called channel code module ${ }^{18}$.

2.3.1 Source Coding: The goal of source coding is efficient convention of source data (input image data) into sequence of bits. The source code reduces the entropy.

2.3.2 Channel: The channel is mathematical model of the medium over which communication occurs.

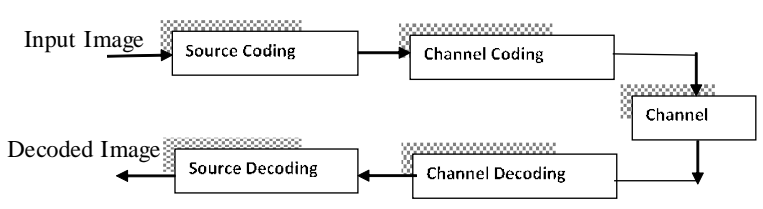

Figure4: Image Compression Scheme [19]

2.3.3 Channel coding: The purpose of channel encoder is to protect the communication system against noise and transmission errors in channel. 


\section{Literature Survey}

3.1 Jonathan Taquet and Claude Labit: "Hierarchical Oriented Predictions for Resolution Scalable Lossless and Near-Lossless Compression of CT and MRI Biomedical Images, Nsme og journsl/conference, Volume/Issue, Page No, Year" ${ }^{10}$.

In this work Authors Presented a new hierarchical approach to resolution scalable, lossless and near-lossless (NLS) compression. It combines the adaptability of DPCM schemes with new hierarchical oriented predictors to provide resolution scalability with better compression performances than the usual hierarchical interpolation predictor or the wavelet transform. Which are dynamically optimized using a least-square criterion. Lossless compression results, which are obtained on a large-scale medical image database, are more than $4 \%$ better on CTs and $9 \%$ better on MRIs than resolution scalable JPEG$2000(\mathrm{~J} 2 \mathrm{~K})$ and close to non scalable CALIC. The HOP algorithm is also well suited for NLS compression, providing an interesting rate-distortion tradeoff compared with JPEGLS and equivalent or a better PSNR than J2K for a high bit rate on noisy (native) medical images and concludes that method shown that, even if providing resolution scalability, some compression improvements could be obtained on noisy native medical images both in lossless and NLS modes compared with the reference algorithms. Some preliminary tests on those images have given promising results.HOP obtained $10 \%$ lossless compression improvements compared with CALIC.

3.2 Sang Heon Lee, Jewoong Ryu and Nam Ik Cho: "Improved H.264/AVC lossless intra compression using multiple partition reduction for 4X4 intra block," 11.

In this work Authors Presented that DPCM (SbS DPCM) is an important prediction technique for the H.264/ AVC lossless intra compression. A new prediction method that is more efficient than the conventional SbS DPCM, thereby improving the overall compression performance. The proposed method prepares 5 partition patterns for each $4 \times 4$ block such as $4 \times 4$ (no partition), $4 \times 2,2 \times 4,2 \times 2$ and $1 \times 1$. The pixels in each partition is intra predicted by SbS DPCM and the best partition which produces minimum bit is selected as the partition pattern for the $4 \times 4$ block. Also, the number of available intra prediction directions is determined according to the partition pattern to avoid too much side-information transmission.

3.3 Saheed Olanigan and Lei Cao: "Multi-Scale Image Compressed Sensing with Optimized Transmission,"12.

In this work Authors Presented that image compressed sensing (CS) often focus on improving the rate- distortion sensing performance but have less consideration of the effect of channel errors on the transmission of CS measurements. Authors explore how the transmission channel errors affect the PSNR performance of the quantized sensing measurements and then increases the resistance of the transmitted data to the noisy channel. We show that the multi-scale block based compressed sensing (MSBCS) using quantization with differential phase code modulation (DPCM), though achieves compression efficiency higher than the regular scalar quantization-based CS, and is more vulnerable to channel errors, and concludes that DPCMbased method in CS though can produce better rate-distortion performance compared to simple SQ-based CS, is more vulnerable to channel errors. Optimal energy allocation methods using both MSE and MAE criteria are proposed and the effect on CS image reconstruction is investigated.

3.4 Ranbeer Tyagi, D. K. Sharma: "Digital Image Compression Comparisons using DPCM and DPCM with LMS Algorithm,"13.

In this work Author Presented that the DPCM and LMS may be used to remove the unused bit in the image for image compression. In this paper Authors compare the compressed image results for 1 and 3 bits DPCM Quantization and DPCM with LMS Algorithm and also compare the histogram, prediction mean square using DPCM Quantization and DPCM with LMS Algorithm for approximately same distortion levels.

Results are presented which show LMS may provide more reduction in transmitted bit rate compared to DPCM when distortion levels are approximately the same for both methods. The results show that the LMS algorithm has the least computational complexity but more reduction in compressed image compare to DPCM with same distortion. The LMS can be used in fixed bit rate environments to decrease the reconstructed Image size and distortion.

\section{PROPOSED METHOD \\ 4.1 Predictive Coding}

Predictive methods involve predicting the current pixel value based on the value of the previously processed pixels. Usually, the difference between the predicted value and the actual value is transmitted. The receiver makes the same prediction as the transmitter and then adds the difference signal in order to reconstruct the original value.

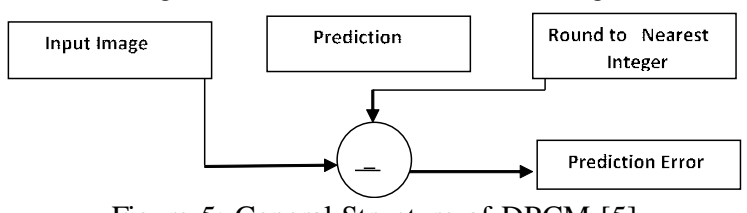

Figure 5: General Structure of DPCM [5] 


\subsection{Mechanism of Algorithm :}

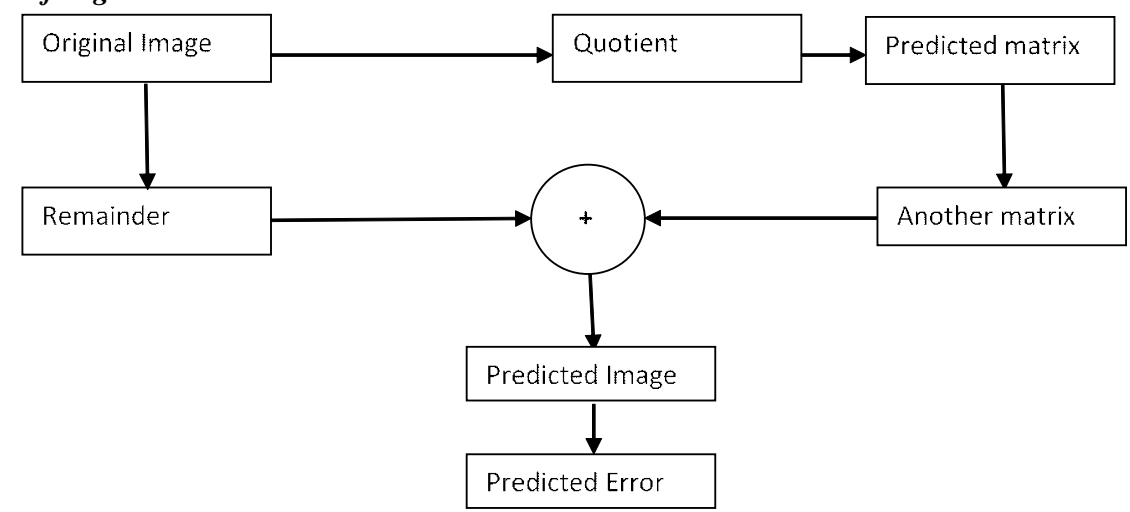

Figure 6: Block Diagram of Mechanism of Algorithm

\section{EXPERIMENTAL RESULTS \& ANALYSIS}

Working Process of Image Compression with Differential Pulse Code Modulation : four Phases:

Image compression technique is very important part in Digital Image Processing. Here we are doing our job in

Input Image

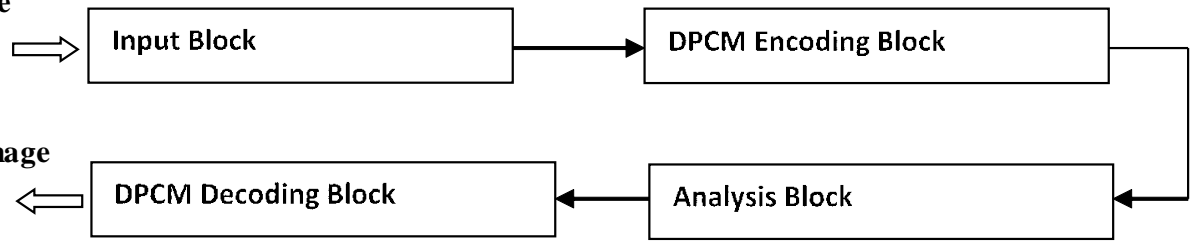

Output Image

Result Analysis

Figure 7: Process Diagram of Our Method

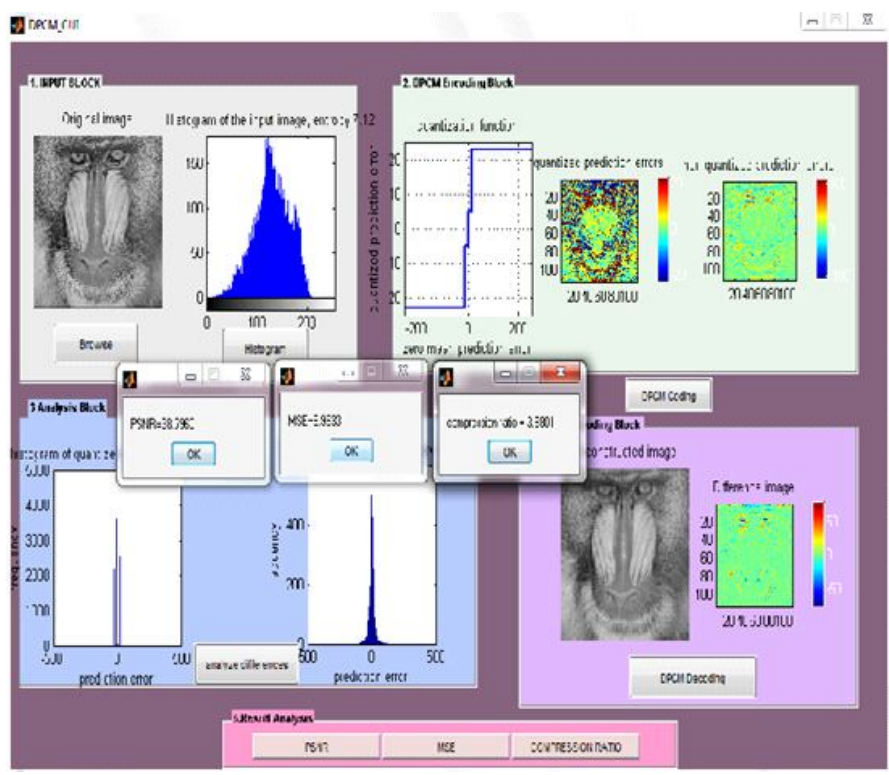

Figure: 8: Overall Analysis of Barbara Image as Input 
In Figure 5.12 we analyse the overall result for image Barbara. In this figure we have 4 blocks each block have different work. By this method we find PSNR, MSE $\&$ CR parameters. Which helps us for further analysis among different algorithm like JPEG 2000, JPEG- LS and many more.

\section{Conclusion}

In this thesis, we introduced a new method of compression which is based on EDT and Huffman entropy encoding. Further, we compare this method with previous JPEG standards such as lossless JPEG and JPEG2000. However compression ratio of the proposed method is more powerful than few previous methods such as lossless JPEG and JPEG2000. This method is suitable for real Time applications. Comparison was based on compression efficiency which is compression ratio and computational complexity. To understand the efficiency of the new method for medical compression and real time application of medical imaging such as telemedicine and online diagnosis, we test our method on medical test cases either.

As it has been proved and illustrated by simulations, the new compression method cause good compression ratio and improves older methods. The compression ratio improvement helps transmission systems to work faster and helps the real time process. It can accelerate transmission time in telemedicine-teleradiology and online diagnosis application. Also it can save data storage space in patient storage of medical images, because it leads more image compression. On the other hand, it is a low complex method in spite of its compression ability. Therefore, it can be efficient for lossless compression and implementation for lossless or near-lossless medical image compression.

\subsection{Future Work}

Our proposed method is suitable for real time application. In near future hardware implementation accelerates the real time application process, suitably satisfied by introducing a low complex and fast algorithm. And our future works would be implementing on hardware and testing images including RGB images. In future, medical image compression technique could be applied on different transforms and the compression percentage could be evaluated.

\section{References}

1. Rafael C. Gonzalez, Richard E. Woods, Steven L. Eddins “Digital Image Processing Using Matlab, Low Price Edition," pp.1-10,374-438, Year of Publication
(2010).

2. S Jayaraman, S Esakkirajan, T Veerakumar "Digital Image Processing, TMH Publication,” pp. 444-542, Year of Publication (2009).

3. Sullivan, B, Ansari, R, Giger, M.L., MacMahon, H., "Relative effects of resolution and quantization on the quality of compressed medical images" pp 987 - 991 vol. 2, Year of Publication (1994).

4. Donald D. Hearn, M. Pauline Baker "Computer Graphics,” pp. 444-542, Year of Publication (2011).

5. Donapati,S.Yagain "A Comparative Study of Effects of CSC on Image Compression Ratios While Using JPEG-XR,"pp 158-161 Year of Publication (2013).

6. Anil K. Jain "Fundamental of Digital Image Processing, PHI Publication," pp. 1-10, 476-560 Year of Publication (2002).

7. Farshid Sepehrband, Mohammad Mortazavi, Seyed Ghorshi and Jeiran Choupan, "Simple Lossless and Near-Lossless Medical Image Compression Based on Enhanced DPCM Transformation," Communications, Computers and Signal Processing (Pac Rim), IEEE Pacific Rim Conference, pp. 62-72, (2011).

8. Sourabh Khire, Lee Cooper, Yuna Park, Alexis Carter, Nikil Jayant, and Joel Saltz, "ZPEG: a hybrid DPCMDCT based approach for compression of Z-stack images," Engineering in Medicine and Biology Society (EMBC), Annual International Conference of the IEEE San Diego, CA, pp. 5424-5427, (2012).

9. Farshid sepehrb, Mohammad Mortazavi, Sayed Ghorshi "Efficient DPCM Predictor for Hardware Implementation of Lossless Medical Brain CT Image Compression," The InternationalConference On Signal AND Electronic System, Poland, September, (2010).

10. Jonathan Taquet, Claude Labit, "Near-Lossless and Scalable Compression for Medical Imaging using A new Adaptive Hierarchical Oriented Prediction" Image Processing (ICIP), 2010 17th IEEE International Conference, pp 481-484, Hong Kong 26 September, (2010).

11. Sang Heon Lee, Jewoong Ryu and Nam Ik Cho "Improved H.264/Avc Lossless Intra Compression Using Multiple Partition Prediction For $4 \times 4$ Intra Block," IEEE International Conference on Image Processing (2011).

12. Saheed Olanigan and Lei Cao "Multi-Scale Image Compressed Sensing With Optimized Transmission," IEEE, Workshop on Signal Processing Systems, (2013).

13. Ranbeer Tyagi, D. K. Sharma "Digital Image Compression Comparisons using DPCM and DPCM 
with LMS Algorithm," International Journal of Computer Applications \& Information Technology Vol. I, Issue II, September (2012).

14. Adina Arthur, V. Saravanan "Efficient Medical Image Compression Technique For Telemedicine Considering Online And Offline Application," Computing, Communication and Applications (ICCCA), . 2012 International Conference Dindigul,Tamilnadu pp. 1-5 Feb (2012).

15. Farshid Sepehrband, Pedram Ghamisi, Ali Mohammadzadeh "Efficient Adaptive Lossless Compression of Hyperspectral Data using Enhanced DPCM," International Journal of Computer Applications Volume 35- No.4, December (2011).

16. Farshid Sepehrband, Mohammad Mortazavi, Seyed Ghorshi and Jeiran Choupan "Simple Lossless and Near-Lossless Medical Image Compression Based on Enhanced DPCM Transformation," Communications, Computers and Signal Processing (PacRim), 2011 IEEE Pacific Rim Conference Victora , BC pp 66-72 Aug (2011).

17. Farshid Sepehrband, Mohammad Mortazavi, Seyed Ghorshi, "An Efficient Lossless Medical Image Transformation Method By Improving Prediction
Model," Signal Processing (ICSP), 2010 IEEE 10th International Conference Beijing, pp 728-731, Oct (2010).

18. Jonathan Taquet and Claude Labit "Hierarchical Oriented Predictions for Resolution Scalable Lossless and Near-Lossless Compression of CT and MRI Biomedical Images," IEEE Transactions On Image Processing, Vol. 21, No. 5, May (2012).

19. Alessia Amelio, Alberto Apostolico, Simona E. Rombo "Image Compression by 2D Motif Basis," IEEE Data Compression Conference (DCC) Snowbird UT pp. 153-162 March (2011).

20. P. Ghamisi, A. Mohammadzadeh, M. R. Sahebi , “ $A$ Novel Real Time Algorithm for Remote Sensing Lossless Data Compression based on Enhanced DPCM", International Journal of Computer Applications Vol. 27, No.1, Aug. (2011).

21. Victor Sanchez, Rafeef Abugharbieh, and Panos Nasiopoulos "3D Scalable Lossless Compression Of Medical Images Based On Global And Local Symmetries," Image Processing (ICIP) 16th IEEE International Conference, Cairo pp. 2525-2528 Nov (2009). 